\title{
Consumo de frutas, legumes e verduras na Região Centro-Oeste do Brasil: prevalência e fatores associados
}

\author{
Consumption of fruit, greens and vegetables in the Midwest region \\ of Brazil: prevalence and associated factors
}

Thaís Fernanda Damiani ${ }^{1}$

Lidia Pitaluga Pereira ${ }^{1}$

Márcia Gonçalves Ferreira ${ }^{1}$

${ }^{1}$ Departamento de Alimentos e Nutrição, Universidade Federal de Mato Grosso. Av. Fernando Corrêa da Costa 2367,

Boa Esperança. 78060-900 Cuiabá MT Brasil. thais.damiani@hotmail.com
Abstract This is a cross-sectional study based on data from the VIGITEL telephone health monitoring survey, aiming to describe the prevalence of adequate fruit, greens and vegetable intake and to identify associated factors. This population-based study included 6696 individuals aged $\geq 18$ years, living in the Midwest of Brazil, in 2012. The adequate consumption of fruit, greens and vegetables (FGV) was the dependent variable and sociodemographic, behavioral and health factors were the independent variables. The prevalence of adequate FGV consumption was $26.3 \%$, being higher among women $(R P a j=1.44 ; C I 95 \%=1.29-1.62)$. The Poisson regression showed that the adequate consumption of FGV was higher among women resident in the Federal District, married and who considered their own health as good/very good. In both genders there was a direct association with education, age and physical activity and inversely associated with the consumption of unhealthy foods. Due to the low prevalence of adequate fruit, greens and vegetable intake in the population of Brazil's Midwest region in 2012, it is proposed that the intervention strategies to increase the consumption of these foods should be encouraged in a global manner.

Key words Food consumption, Fruit, Vegetables, Epidemiological surveys, Data systems
Resumo Estudo transversal, baseado em dados do sistema de monitoramento por inquérito telefônico - VIGITEL, com o objetivo de estimar a prevalência e os fatores associados ao consumo recomendado de frutas, legumes e verduras (FLV) em 6696 indivíduos com idade $\geq 18$ anos, de ambos os sexos, residentes na Região Centro-Oeste do Brasil, em 2012. O consumo recomendado foi a variável dependente e variáveis sociodemográficas, comportamentais e relacionadas à saúde foram as independentes. A prevalência do consumo recomendado de FLV foi de 26,3\%, sendo maior entre as mulheres (RPaj $=1,44 ; I C 95 \%=$ 1,29-1,62). A regressão de Poisson entre o desfecho e as variáveis independentes mostrou maior prevalência do consumo recomendado entre as mulheres residentes no Distrito Federal, casadas e que consideravam seu estado de saúde como bom/ muito bom. Em ambos os sexos, observou-se associação direta do desfecho com a escolaridade, idade e prática de atividade física e associação inversa com o consumo de alimentos não saudáveis. Devido à baixa prevalência do consumo recomendado de FLV na população da Região Centro-Oeste do Brasil no ano de 2012, propõe-se que as estratégias de intervenção para melhorar o consumo desses alimentos sejam feitas de forma globalizada.

Palavras-chave Consumo de alimentos, Frutas, Verduras, Inquéritos epidemiológicos, Sistemas de informação 


\section{Introdução}

Segundo a Pesquisa Nacional por Amostra de Domicílios (PNAD), realizada em 2012, a Região Centro-Oeste apresenta o maior fluxo migratório do Brasil. Cerca de 34,1\% dos habitantes do Centro-Oeste nasceram em outras regiões do país, com destaque para o estado de Mato Grosso e para o Distrito Federal ${ }^{1}$. O aumento do volume de investimentos em infraestrutura e a expansão da fronteira agrícola nessa região possibilitou o fortalecimento e ampliou ainda mais o fluxo migratório ${ }^{1}$. O avanço da economia e do agronegócio do Centro-Oeste teve impacto positivo nos indicadores sociais do Índice de Desenvolvimento Humano (IDH). Entre 1991 e 2010, os quatro Estados da região registraram crescimento médio de $49 \%$ no IDH e avançaram no ranking nacional ${ }^{2}$.

A Região Centro-Oeste é responsável por $41 \%$ da produção agrícola nacional, porém a produção de frutas ainda é incipiente ${ }^{3}$. Diante desse cenário e do aumento da prevalência de excesso de peso e obesidade na região, especialmente em Cuiabá, pesquisas científicas e tecnológicas precisam ser ampliadas para a geração e a difusão de novos conhecimentos que possibilitem o desenvolvimento social e de politicas públicas com benefícios diretos à sociedade 4 .

Frutas, legumes e verduras (FLV) são componentes importantes de uma alimentação saudável e seu consumo adequado é um dos principais fatores de proteção para as doenças crônicas não transmissíveis (DCNT) ${ }^{5}$. De acordo com a Organização Mundial da Saúde (OMS) ${ }^{6}$, o baixo consumo desses alimentos está entre os dez principais fatores de risco para a carga total de doença em todo o mundo, sendo responsável por aproximadamente 2,7 milhões de óbitos anualmente.

As FLV devem compor uma dieta saudável, pois são fontes de micronutrientes, fibras e de outros componentes com propriedades funcionais ${ }^{5}$. A baixa densidade energética desse grupo em relação ao volume do alimento consumido favorece a manutenção do peso corporal saudável ${ }^{7}$.

Segundo recomendações da Organização das Nações Unidas sobre Alimentos e Agricultura (Food and Agriculture Organization of the United Nations - FAO) e da $\mathrm{OMS}^{6}$, o consumo mínimo de FVL deve ser de $400 \mathrm{~g} /$ dia ou o equivalente a cinco porções. O Guia Alimentar para a População Brasileira enfatiza que a alimentação deve ser baseada em alimentos frescos (frutas, carnes, legumes) e minimamente processados (arroz, feijão e frutas secas), contraindicando produtos ultraprocessados ${ }^{8}$. Tal recomendação visa reduzir o risco de desenvolvimento de DCNT e também a prevenção e a correção de várias deficiências de micronutrientes, principalmente nos países menos desenvolvidos ${ }^{6}$.

A promoção do consumo médio de FLV em nível populacional tornou-se uma prioridade em saúde pública em vários países na última década. No entanto, no Brasil e em diversos outros países, evidências sugerem que o consumo de FLV está substancialmente abaixo da recomendação da $\mathrm{OMS}^{4,9}$. A ingestão desses alimentos varia consideravelmente entre os países, sendo menor nas regiões menos desenvolvidas, refletindo as condições socioeconômicas, culturais e agrícolas ${ }^{10}$.

No Brasil, estima-se que o consumo de FLV corresponda a menos da metade das recomendações nutricionais, sendo ainda mais deficitário entre as famílias de baixa renda ${ }^{11}$. Dados da Pesquisa de Orçamentos Familiares (POF), em 2008-2009, concluíram que mais de 90\% da população brasileira têm ingestão abaixo dos níveis recomendados pelo Ministério da Saúde ${ }^{12}$. Dados do Sistema de Vigilância de Fatores de Risco e Proteção para Doenças Crônicas por Inquérito Telefônico (VIGITEL) também indicaram baixa frequência de consumo recomendado de FLV na maioria das cidades estudadas, variando entre 16\% em Rio Branco e 30,3\% em Florianópolis ${ }^{4,13}$.

Nesse sentido, o objetivo deste estudo é estimar a prevalência e identificar os fatores associados ao consumo adequado de FLV na população com 18 anos ou mais de idade da Região Centro-Oeste do Brasil, visando contribuir com informações para o planejamento de intervenções direcionadas à população com risco de baixo consumo de FLV.

\section{Métodos}

Estudo com delineamento transversal, de base populacional, que utilizou dados individuais do Sistema de Vigilância de Fatores de Risco e Proteção para Doenças Crônicas (VIGITEL), obtidos por meio do Ministério da Saúde, para a Região Centro-Oeste do Brasil, no ano de 2012.

O VIGITEL é um sistema de monitoramento contínuo da frequência e distribuição de fatores de risco e proteção das doenças crônicas não transmissíveis. Implantado pelo Ministério da Saúde, em 2006, nas 26 capitais brasileiras e no Distrito Federal, utiliza amostras probabilísticas da população de adultos, providos por, pelo menos, uma linha de telefone fixa no ano vigente do estudo. Os procedimentos de amostragem 
empregados estabelecem o mínimo de 2.000 indivíduos com 18 anos ou mais de idade em cada cidade como o tamanho amostral, para que se possa estimar com coeficiente de confiança de 95\% e erro máximo de cerca de dois pontos percentuais, a frequência de qualquer fator de risco na população adulta ${ }^{4}$.

Neste estudo foram analisados os dados de indivíduos com idade $\geq 18$ anos, de ambos os sexos e residentes na Região Centro-Oeste do Brasil. No ano de 2012, o VIGITEL realizou 6.742 entrevistas completas nessa região entre os meses de julho de 2012 e fevereiro de 2013, sendo 1.666 em Campo Grande, 1.666 em Cuiabá, 1.710 em Goiânia e 1.700 em Brasília. A duração média da entrevista foi de 11 minutos, a taxa média de sucesso foi de $64,9 \%$ e a taxa de recusa foi de $5,9 \%{ }^{3}$. Foram excluídas 46 mulheres que declararam gravidez na época da entrevista. Após a exclusão, o consumo recomendado de frutas, legumes e verduras (FLV) foi avaliado em um total de 6.696 indivíduos.

A variável dependente (desfecho) foi a prevalência de consumo recomendado de FLV. No Sistema VIGITEL essa variável é identificada por "flvreco", referindo-se ao consumo diário de cinco ou mais porções desses alimentos, conforme preconizado pela Organização Mundial da Saúde. A quantidade de porções de frutas e hortaliças consumidas habitualmente foi estimada pelo Vigitel a partir de questões sobre a quantidade de frutas ou sucos de frutas consumidos por dia e sobre o hábito de consumir hortaliças cruas (na forma de saladas) ou cozidas, no almoço e no jantar. Essas questões foram feitas apenas para indivíduos que informam consumir frutas e hortaliças em cinco ou mais dias da semana. O cômputo do total diário de porções foi feito considerando-se cada fruta ou cada suco de fruta como equivalente a uma porção. Entretanto, para assegurar a necessária diversificação da dieta, limita-se em três o número máximo de porções diárias computado para frutas e em um para sucos. No caso de hortaliças, computa-se um número máximo de quatro porções diárias, situação que caracteriza indivíduos que informam ter o hábito de consumir hortaliças cruas e hortaliças cozidas no almoço e no jantar 4 .

As variáveis independentes selecionadas para este estudo foram divididas em três blocos: sociodemográficas (idade, sexo, estado civil, raça/ cor, nível de escolaridade e situação de trabalho), de estilo de vida (consumo/consumo abusivo de bebida alcoólica, consumo de feijão, carne com excesso de gordura, pele de frango, leite integral, refrigerante, doce; uso/quantidade de cigarro; prática de exercícios físicos, atividades físicas no lazer e/ou no deslocamento) e aquelas relacionadas à saúde (classificação do peso e autoavaliação do estado de saúde).

A raça/cor foi autorreferida e classificada em cinco categorias: branca, preta, parda, amarela e indígena. Neste estudo, essa variável foi agrupada em duas categorias: branca e não branca. A situação conjugal foi agrupada em casado, solteiro e outros (viúvo, em união estável, separado ou divorciado). A escolaridade foi considerada em anos de estudo, utilizando-se as seguintes categorias nas análises: $\leq 8$ anos, $9-11$ anos e $\geq 12$. A variável denominada "trabalho" refere-se a alguma atividade laboral exercida nos últimos três meses $^{4}$.

O consumo de bebidas alcoólicas foi classificado em sim ou não, de acordo com a informação do entrevistado. Para medir o consumo abusivo de bebidas alcoólicas considerou-se a ingestão de quatro ou mais doses para as mulheres e cinco ou mais para os homens, em uma mesma ocasião, nos últimos 30 dias. A dose de bebida alcoólica foi avaliada por uma lata de cerveja, uma taça de vinho ou uma dose de bebida destilada ${ }^{4}$.

Com relação à atividade física, os indivíduos foram classificados em praticantes ou não, de acordo com o relato da prática de exercício físico nos últimos três meses. O cálculo deste indicador foi obtido pela divisão do número de indivíduos que praticaram pelo menos 150 minutos semanais de atividade física de intensidade leve ou moderada, ou pelo menos 75 minutos semanais de intensidade vigorosa, pelo número de indivíduos entrevistados ${ }^{11}$. A atividade física no deslocamento investigou se os indivíduos faziam algum trajeto a pé ou de bicicleta enquanto se deslocavam para o trabalho ou escola, com dispêndio de pelo menos 30 minutos diários no percurso de ida e volta ${ }^{4,14}$.

O Índice de Massa Corporal (IMC) foi utilizado para classificar o peso dos participantes do estudo, respeitando-se as faixas etárias, de acordo com as recomendações da Organização Mundial da Saúde: adolescentes (18-19 anos), adultos (2059 anos) e idosos ( $\geq 60$ anos). Para os adolescentes, o IMC foi expresso em z-escore avaliado segundo sexo e idade, sendo classificados em: baixo peso $(<-2)$; eutrofia $(\geq-2 \mathrm{e} \leq+1)$; sobrepeso ( $>$ +1 e $\leq+2)$ e obesidade $(>+2)^{15}$. Para adultos os três pontos de corte de IMC $\left(\mathrm{kg} / \mathrm{m}^{2}\right)$ utilizados foram $<18,5$ - baixo peso; $\geq 18,5$ e $<25,0$ - eutrófico; $\geq 25,0$ e $<30$ - sobrepeso e $\geq 30,0$ - obesida$\mathrm{de}^{16}$. Os pontos de corte do IMC $\left(\mathrm{kg} / \mathrm{m}^{2}\right)$ utiliza- 
dos para classificar os idosos foram: $<22-$ baixo peso; $22-27$ - eutrófico e $>27$ - sobrepeso $^{17}$.

A variável autoavaliação do estado de saúde foi reagrupada nesse estudo, de acordo com a frequência de citação das respostas, considerando-se nas análises as seguintes categorias: ruim/ muito ruim, regular, bom e muito bom ${ }^{4}$.

As análises foram ponderadas de acordo com as diferenças na composição sociodemográfica da amostra do VIGITEL em relação à composição da população total com idade $\geq 18$ anos de cada cidade, segundo a distribuição censitária do ano 2010. Os dados foram processados com auxílio do software Stata versão 11, com a aplicação dos fatores de ponderação, através do comando survey. A distribuição da população para as variáveis independentes de interesse do estudo foi apresentada em percentual.

Estimou-se a prevalência $(\mathrm{P})$, razão de prevalência bruta (RP) e seus respectivos intervalos de confiança (IC 95\%) na análise bivariada. As variáveis que apresentaram valor $\mathrm{p} \leq 0,20$ na análise bivariada foram selecionadas para a análise múltipla. A regressão de Poisson foi utilizada para estimar a razão de prevalência ajustada (RPaj) com seus respectivos intervalos de confiança (IC 95\%). As variáveis independentes foram ajustadas por idade e escolaridade. O nível de significância adotado foi de $5 \%$.

Este estudo utilizou exclusivamente dados secundários, provenientes do sistema VIGITEL, como resposta a uma solicitação prévia de dados individuais. O VIGITEL foi submetido à aprovação do Conselho Nacional de Saúde por meio da Comissão Nacional de Ética em Pesquisa para Seres Humanos do Ministério da Saúde. O consentimento livre e esclarecido foi substituído pelo consentimento verbal, obtido oralmente no momento do contato telefônico, por se tratar de um estudo realizado por entrevistas telefônicas. O projeto de pesquisa deste estudo foi aprovado pelo Comitê de Ética do Hospital Universitário Júlio Muller.

\section{Resultados}

Foram analisados dados de 6.696 indivíduos, sendo $52,6 \%(\mathrm{n}=3522)$ do sexo feminino, com média de idade de 45,1 anos e desvio padrão de 16,8 anos. Entre os participantes $41,5 \%$ ( $\mathrm{n}=$ 2778) eram casados, $40,6 \%(\mathrm{n}=2718)$ da raça/ cor branca, $69,4 \%(n=4647)$ estavam empregados e $38,2 \%(n=2557)$ relataram de 9 a 11 anos de estudo. Apenas 26,3\% $(\mathrm{n}=1761)$ dos indiví- duos consumiram frutas e hortaliças em cinco ou mais porções diárias, conforme a recomendação.

A prevalência do consumo recomendado de frutas, legumes e verduras apresentou diferença segundo o sexo, sendo maior entre as mulheres $(\mathrm{RPaj}=1,44$; IC $95 \%=1,29-1,62)$. As associações observadas na análise bivariada mantiveram-se na análise múltipla, com exceção da perda de associação com as variáveis raça/cor e tabagismo para os homens e raça/cor, tabagismo, consumo de leite integral e consumo de doces para as mulheres.

$\mathrm{Na}$ análise múltipla, entre os homens, a variável sociodemográfica que mostrou associação direta com o consumo recomendado de FLV foi a escolaridade, sendo maior para aqueles com 9 -11 anos de estudo (RPaj = 1,67; IC 95\%: 1,23 $-2,28)$ e com mais de 12 anos de estudo (RPaj $=2,54$; IC 95\%: 1,88-3,44), em relação aos que estudaram até 8 anos. Houve associação da idade com o consumo recomendado de FLV apenas para os homens com idade $\geq 65$ anos (Tabela 1). Para as mulheres, as variáveis que se associaram com o desfecho avaliado foram: idade de $45-54$ anos $(\mathrm{RPaj}=1,59$; IC 95\% 1,27 - 1,99), 55-64 anos $(\mathrm{RPaj}=1,43$; IC95\% $1,12-1,82)$ e $\geq 65$ anos $(\mathrm{RPaj}=1,47$; IC95\% 1,14-1,89) ; escolaridade de 9-11 anos de estudo ( $\mathrm{RPaj}=1,39$; IC 95\%: $1,17-1,65)$ e 12 ou mais $(\mathrm{RPaj}=1,97$; IC 95\%: 1,67 - 2,33). As mulheres do Distrito Federal apresentaram maior prevalência do consumo recomendado de FLV (RPaj = 1,35; IC 95\%: 1,16 $-1,58)$ em relação às residentes em Cuiabá. Entre as mulheres casadas (RPaj $=1,23$; IC 95\%: 1,05 $-1,44)$ observou-se maior prevalência de consumo recomendado de FLV em relação às solteiras (Tabela 2).

Entre os homens, verificou-se associação direta do consumo recomendado de FLV com a prática de exercícios físicos nos últimos 3 meses e com a atividade física no lazer/transporte $(\mathrm{RPaj}=$ 1,92; IC 95\%: 1,52 - 2,42 e RPaj = 1,72; IC 95\%: $1,39-2,12$ ), respectivamente (Tabela 3$)$. Entre as mulheres houve maior prevalência de consumo recomendado de FLV para as que praticaram exercícios físicos nos 3 meses que antecederam a entrevista e entre as que relataram prática de atividade física no lazer/transporte $(\mathrm{RPaj}=1,65$; IC 95\%: 1,46 - 1,88 e RPaj = 1,50; IC 95\%: 1,331,70 ), respectivamente (Tabela 4 ).

As variáveis do consumo alimentar que se associaram com o consumo recomendado de FLV foram as mesmas para ambos os sexos, exceto para o consumo regular de feijão (Tabelas 3 e 4). A prevalência do desfecho avaliado foi maior para 
Tabela 1. Características descritivas da população (\%), prevalência de consumo recomendado de frutas, legumes e verduras (P\%), razão de prevalência bruta (RP) e ajustada (RPaj) e intervalo de confiança de 95\% (IC 95\%), segundo variáveis sociodemográficas para o sexo masculino, Região Centro-Oeste, VIGITEL, 2012.

\begin{tabular}{|c|c|c|c|c|c|c|c|}
\hline Variáveis & $\mathbf{n}$ & $\%$ & $\mathbf{P}(\%)$ & RP (IC 95\%) & p-valor & RPaj(IC 95\%) & p-valor \\
\hline \multicolumn{8}{|l|}{ Idade $(\operatorname{anos})^{*}$} \\
\hline$\leq 24$ & 378 & 20,6 & 17,9 & 1 & & 1 & \\
\hline $25-34$ & 458 & 26,4 & 24,1 & $1,34(0,97-1,86)$ & 0,078 & $1,31(0,95-1,82)$ & 0,096 \\
\hline $35-44$ & 567 & 20,7 & 20,9 & $1,17(0,85-1,60)$ & 0,337 & $1,30(0,95-1,78)$ & 0,094 \\
\hline $45-54$ & 487 & 15,4 & 17,2 & $0,96(0,68-1,35)$ & 0,818 & $1,17(0,83-1,64)$ & 0,369 \\
\hline $55-64$ & 383 & 9,1 & 19,6 & $1,09(0,77-1,55)$ & 0,626 & $1,41(0,98-2,01)$ & 0,090 \\
\hline$\geq 65$ & 352 & 7,8 & 26,7 & $1,49(1,06-20,9)$ & 0,021 & $2,02(1,42-2,87)$ & $<0,01$ \\
\hline \multicolumn{8}{|l|}{ Raça/cor ${ }^{\dagger}$} \\
\hline Outras & 1410 & 60,5 & 18,6 & 1 & & 1 & \\
\hline Branca & 1031 & 39,5 & 25,1 & $1,35(1,10-1,65)$ & 0,004 & $1,20(0,98-1,47)$ & 0,081 \\
\hline \multicolumn{8}{|c|}{ Escolaridade (anos) ${ }^{* *}$} \\
\hline $0-8$ & 815 & 35,1 & 13,5 & 1 & & 1 & \\
\hline $9-11$ & 995 & 39,0 & 20,6 & $1,53(1,15-2,02)$ & 0,003 & $1,67(1,23-2,28)$ & $<0,01$ \\
\hline 12 e mais & 815 & 25,9 & 31,4 & $2,33(1,77-3,08)$ & $<0,01$ & $2,54(1,88-3,44)$ & $<0,01$ \\
\hline \multicolumn{8}{|l|}{ Estado Civil ${ }^{\dagger}$} \\
\hline Solteiro & 797 & 41,0 & 20,0 & 1 & & 1 & \\
\hline Casado & 1329 & 43,3 & 22,3 & $1,11(0,90-1,39)$ & 0,332 & $1,21(0,91-1,59)$ & 0,404 \\
\hline Outros & 484 & 15,7 & 18,2 & $0,91(0,67-1,23)$ & 0,545 & $1,05(0,75-1,47)$ & 0,939 \\
\hline \multicolumn{8}{|l|}{ Cidade $^{\dagger}$} \\
\hline Cuiabá & 649 & 10,7 & 19,2 & 1 & & 1 & \\
\hline Campo Grande & 658 & 15,2 & 17,5 & $0,91(0,69-1,20)$ & 0,519 & $0,92(0,70-1,21)$ & 0,552 \\
\hline Goiânia & 645 & 25,5 & 22,2 & $1,15(0,89-1,49)$ & 0,278 & $1,13(0,88-1,47)$ & 0,397 \\
\hline Distrito Federal & 673 & 48,6 & 21,6 & $1,13(0,87-1,45)$ & 0,360 & $1,07(0,83-1,38)$ & 0,663 \\
\hline \multicolumn{8}{|l|}{ Trabalha $^{\dagger}$} \\
\hline Sim & 2068 & 80,9 & 20,6 & 1 & & 1 & \\
\hline Não & 557 & 19,1 & 22,1 & $1,07(0,84-1,36)$ & 0,568 & $1,11(0,86-1,42)$ & 0,447 \\
\hline
\end{tabular}

${ }^{*} \mathrm{RP}$ ajustada por escolaridade. ${ }^{* *} \mathrm{RP}$ ajustada por idade. ${ }^{\dagger} \mathrm{RP}$ ajustada por idade e escolaridade.

os homens (RPaj = 1,35; IC 95\%: 1,11 - 1,66) e mulheres $(\mathrm{RPaj}=1,34$; IC 95\%: 1,14 - 1,57) que não relataram consumo de carne com excesso de gordura ; entre os homens ( $\mathrm{RPaj}=1,42$; IC 95\%: $1,12-1,81)$ e mulheres $(\mathrm{RPaj}=1,26$; IC $95 \%$ : $1,02-1,56)$ que não consumiam frango com pele e para homens (RPaj $=2,42$; IC 95\%: 1,85-3,18) e mulheres $(\mathrm{RPaj}=1,81$; IC 95\%: 1,45-2,26) que referiram não consumir refrigerantes ou sucos artificiais em cinco ou mais dias da semana. O consumo de feijão em cinco ou mais dias da semana mostrou associação com o consumo recomendado de FLV para os homens ( $\mathrm{RPaj}=1,33$; IC 95\%: $1,01-1,74)$ e associação marginal para as mulheres (RPaj = 1,12; IC 95\%: 0,98-1,29).

A variável estado de saúde autorreferida mostrou associação com o consumo recomendado de FLV apenas para o sexo feminino. Mulheres que autorrelataram seu estado de saúde como Bom
$(\mathrm{RPaj}=1,48$, IC 95\% 1,04-2,10) e Muito Bom $(\mathrm{RPaj}=1,93$, IC 95\% 1,35-2,78) apresentaram maior prevalência do consumo de FLV, comparadas àquelas que o referiram como muito ruim. A variável classificação de peso não apresentou associação com o desfecho avaliado em ambos os sexos (Tabela 4).

\section{Discussão}

Os resultados deste estudo mostraram que cerca de 3/4 da população da Região Centro-Oeste do Brasil não atenderam à recomendação mínima de consumo diário de 5 ou mais porções de frutas, legumes e verduras no ano de 2012, conforme preconiza a $\mathrm{OMS}^{6}$. A prevalência de consumo recomendado de FLV foi $44 \%$ maior entre as mulheres. Em ambos os sexos, observou- 
Tabela 2. Características descritivas da população (\%), prevalência de consumo recomendado de frutas, legumes e verduras (P\%), razão de prevalência bruta (RP) e ajustada (RPaj) e intervalo de confiança de 95\% (IC 95\%), segundo variáveis sociodemográficas para o sexo feminino, Região Centro-Oeste, VIGITEL, 2012.

\begin{tabular}{|c|c|c|c|c|c|c|c|}
\hline Variáveis & $\mathbf{n}$ & $\%$ & $\mathbf{P}(\%)$ & RP (IC 95\%) & p-valor & RPaj(IC 95\%) & p-valor \\
\hline \multicolumn{8}{|l|}{${\text { Idade }(\text { anos })^{*}}^{*}$} \\
\hline$\leq 24$ & 373 & 14,3 & 27,8 & 1 & & 1 & \\
\hline $25-34$ & 676 & 27,1 & 28,9 & $1,04(0,81-1,34)$ & 0,762 & $1,07(0,84-1,36)$ & 0,601 \\
\hline $35-44$ & 817 & 21,5 & 31,8 & $1,14(0,90-1,45)$ & 0,272 & $1,27(1,01-1,60)$ & 0,040 \\
\hline $45-54$ & 807 & 17,2 & 37,2 & $1,34(1,06-1,69)$ & 0,014 & $1,59(1,27-1,99)$ & $<0,01$ \\
\hline $55-64$ & 706 & 11,3 & 31,6 & $1,14(0,89-1,45)$ & 0,310 & $1,43(1,12-1,82)$ & 0,004 \\
\hline$\geq 65$ & 692 & 8,6 & 29,9 & $1,07(0,84-1,38)$ & 0,568 & $1,47(1,14-1,89)$ & 0,003 \\
\hline \multicolumn{8}{|l|}{$\operatorname{Raça} / \operatorname{cor}^{\dagger}$} \\
\hline Outras & 2006 & 58,5 & 29,2 & 1 & & 1 & \\
\hline Branca & 1729 & 41,5 & 34,8 & $1,19(1,05-1,35)$ & 0,007 & $1,11(0,98-1,26)$ & 0,125 \\
\hline \multicolumn{8}{|c|}{ Escolaridade $(\text { anos })^{* *}$} \\
\hline $0-8$ & 1323 & 33,5 & 23,6 & 1 & & 1 & \\
\hline $9-11$ & 1475 & 37,5 & 29,6 & $1,25(1,06-1,48)$ & 0,008 & $1,39(1,17-1,65)$ & $<0,01$ \\
\hline 12 e mais & 1273 & 29,0 & 41,9 & $1,77(1,52-2,08)$ & $<0,01$ & $1,97(1,67-2,33)$ & $<0,01$ \\
\hline \multicolumn{8}{|l|}{ Estado Civil ${ }^{\dagger}$} \\
\hline Solteiro & 1085 & 34,7 & 28,8 & 1 & & 1 & \\
\hline Casado & 1637 & 39,9 & 35,2 & $1,22(1,05-1,42)$ & 0,008 & $1,23(1,05-1,44)$ & 0,016 \\
\hline Outros & 1324 & 25,4 & 28,0 & $0,97(0,82-1,16)$ & 0,757 & $1,04(0,86-1,25)$ & 0,704 \\
\hline \multicolumn{8}{|l|}{ Cidade $^{\dagger}$} \\
\hline Cuiabá & 1005 & 10,3 & 25,4 & 1 & & 1 & \\
\hline Campo Grande & 998 & 14,9 & 28,0 & $1,10(0,93-1,31)$ & 0,275 & $1,14(0,96-1,36)$ & 0,116 \\
\hline Goiânia & 1052 & 25,7 & 29,5 & $1,16(0,99-1,36)$ & 0,073 & $1,17(0,99-1,37)$ & 0,063 \\
\hline Distrito Federal & 1016 & 49,1 & 34,2 & $1,34(1,15-1,58)$ & $<0,01$ & $1,35(1,16-1,58)$ & $<0,01$ \\
\hline \multicolumn{8}{|l|}{ Trabalha $^{\dagger}$} \\
\hline Sim & 2207 & 59,0 & 30,9 & 1 & & 1 & \\
\hline Não & 1864 & 41,0 & 31,5 & $1,02(0,90-1,15)$ & 0,779 & $1,13(0,99-1,30)$ & 0,095 \\
\hline
\end{tabular}

${ }^{\star} \mathrm{RP}$ ajustada por escolaridade. ${ }^{* *} \mathrm{RP}$ ajustada por idade. ${ }^{\dagger} \mathrm{RP}$ ajustada por idade e escolaridade.

se associação direta da variável desfecho com o nível de escolaridade, idade e com a prática de atividade física e, relação inversa com o consumo de alimentos não saudáveis. Para as mulheres observou-se maior prevalência do consumo recomendado de FLV entre as casadas, residentes no Distrito Federal e que autorrelataram seu estado de saúde como bom ou muito bom.

A prevalência de consumo recomendado de FLV encontrada para a Região Centro-Oeste neste estudo $(26,3 \%)$ foi superior àquela observada nacionalmente pelo Vigitel no mesmo ano $(22,7 \%)^{6}$ e pelo Behavioral Risk Factor Surveillance System (BRFSS) nos Estados Unidos (23,4\%,) em 200918. Em 2011, ocorreram alterações na pesquisa BRFFS, inclusive nas questões utilizadas para avaliar o consumo de FLV, dificultando a comparação com os dados deste estudo ${ }^{19}$. Dados do VIGITEL 2008-2010 analisados pelo Observatório em Iniquidades em Saúde mostraram a Região Centro-Oeste com o maior crescimento no consumo per capita desses alimentos ${ }^{20}$. Da mesma forma, resultados da POF 2008-2009 evidenciaram tal crescimento com relação à disponibilidade desses alimentos nos domicílios da região ${ }^{12}$.

Os resultados do presente estudo concordam com evidências de diferentes pesquisas no que concerne a maior frequência de consumo recomendado de FLV entre as mulheres ${ }^{21-24}$. Mulheres residentes no Distrito Federal apresentaram maior prevalência de consumo comparadas àquelas residentes em outras capitais da região. Dados do Vigitel de 2012 apontam o Distrito Federal como a terceira maior frequência encon- 
Tabela 3. Prevalência de consumo recomendado de frutas, legumes e verduras (P\%), razão de prevalência bruta (RP) e ajustada (RPaj) e intervalo de confiança de 95\% (IC 95\%), segundo variáveis do estilo de vida e relacionadas à saúde para o sexo masculino, Região Centro-Oeste, VIGITEL, 2012.

\begin{tabular}{|c|c|c|c|c|c|c|c|}
\hline Variáveis & $\mathbf{n}$ & $\%$ & $\mathrm{P}(\%)$ & RP (IC 95\%) & p-valor & RPaj(IC 95\%)* & p-valor \\
\hline \multicolumn{8}{|c|}{ Consumo de bebida alcoólica } \\
\hline Sim & 1335 & 50,5 & 21,3 & 1 & & 1 & \\
\hline Não & 1290 & 49,5 & 20,5 & $1,04(0,85-1,27)$ & 0,685 & $1,00(0,82-1,21)$ & 0,960 \\
\hline \multicolumn{8}{|c|}{$\begin{array}{l}\text { Consumo abusivo de bebidas } \\
\text { alcoólicas }\end{array}$} \\
\hline Sim & 708 & 28,1 & 18,2 & 1 & & 1 & \\
\hline Não & 1917 & 71,9 & 21,9 & $1,20(0,95-1,52)$ & 0,116 & $1,24(0,99-1,57)$ & 0,062 \\
\hline \multicolumn{8}{|l|}{ Exercício Físico (3 meses) } \\
\hline Não & 1097 & 40,9 & 12,4 & 1 & & 1 & \\
\hline Sim & 1528 & 59,1 & 26,8 & $2,16(1,72-2,71)$ & $<0,01$ & $1,92(1,52-2,42)$ & $<0,01$ \\
\hline \multicolumn{8}{|c|}{ Atividade física no lazer/transporte } \\
\hline Não & 1332 & 47,6 & 14,7 & 1 & & 1 & \\
\hline Sim & 1293 & 52,4 & 26,5 & $1,80(1,46-2,22)$ & $<0,01$ & $1,72(1,39-2,12)$ & $<0,01$ \\
\hline \multicolumn{8}{|l|}{ Fuma } \\
\hline Sim & 335 & 13,2 & 13,5 & 1 & & 1 & \\
\hline Não & 2290 & 86,8 & 22,0 & $1,63(1,13-2,36)$ & 0,009 & $1,43(0,99-2,07)$ & 0,050 \\
\hline \multicolumn{8}{|l|}{ Cigarros/dia } \\
\hline $1-4$ & 30 & 8,9 & 20,1 & 1 & & 1 & \\
\hline $5-9$ & 44 & 13,3 & 24,7 & $1,23(0,39-3,87)$ & 0,727 & $1,16(0,40-3,38)$ & 0,496 \\
\hline $10-19$ & 83 & 32,5 & 15,5 & $0,77(0,27-2,18)$ & 0,622 & $0,82(0,31-2,22)$ & 0,947 \\
\hline$\geq 20$ & 122 & 45,3 & 5,6 & $0,28(0,09-0,89)$ & 0,031 & $0,31(0,10-0,94)$ & 0,063 \\
\hline
\end{tabular}

trada para o consumo de FLV no país $(34,2 \%)$ entre o sexo feminino ${ }^{4}$. Uma possível explicação para esse achado é a maior renda per capita observada na capital federal comparada às demais capitais da região. Segundo o Instituto de Pesquisa Econômica Aplicada (IPEA), grande parte dos indicadores sociais do Distrito Federal está melhor que a média brasileira. A renda domiciliar é a maior no país, assim como o número de anos de estudo da população residente. A renda mais alta resulta em melhores níveis de educação, em maior expectativa de vida e na qualidade da alimentação ${ }^{25}$.

A escolaridade foi o indicador sociodemográfico utilizado para estimar diferenças sociais entre os indivíduos deste estudo, pois dados sobre renda e poder de compra não estão disponíveis no sistema VIGITEL. Homens e mulheres com maior nível de escolaridade apresentaram maior frequência de consumo recomendado de frutas, legumes e verduras. A escolaridade influencia a escolha de alimentos saudáveis, pois pode significar acesso a melhores oportunidades de emprego, renda e informação ${ }^{26}$.

Estudos internacionais observaram maior consumo de frutas, legumes e verduras em indivíduos de maior escolaridade de ambos os $\operatorname{sexos}^{27,28}$. Resultados semelhantes foram encontrados no Brasil ${ }^{21,29}$. Um estudo desenvolvido em Belém (PA) por Moura et al. ${ }^{22}$ mostrou que a chance de consumo recomendado de FLV foi 3,1 vezes maior para os homens que estudaram 12 anos ou mais, comparados com aqueles com escolaridade de até oito anos. Para as mulheres, embora essa diferença não tenha sido detectada após ajuste para ocupação, observou-se tendência de maior consumo naquelas com maior escolaridade.

No presente estudo, a idade também associou-se ao consumo recomendado de FLV para 
Tabela 3. continuação

\begin{tabular}{|c|c|c|c|c|c|c|c|}
\hline Variáveis & $\mathbf{n}$ & $\%$ & $\begin{array}{c}\mathbf{P} \\
(\%)\end{array}$ & RP (IC 95\%) & p-valor & $\operatorname{RPaj}(\mathrm{IC} 95 \%)^{*}$ & p-valor \\
\hline \multicolumn{8}{|l|}{ Consumo de feijão } \\
\hline Não & 471 & 15,8 & 18,1 & 1 & & 1 & \\
\hline $\operatorname{Sim}$ & 2154 & 84,2 & 21,4 & $1,18(0,90-1,57)$ & 0,230 & $1,33(1,01-1,74)$ & 0,032 \\
\hline \multicolumn{8}{|c|}{$\begin{array}{l}\text { Consumo carne com excesso } \\
\text { de gordura }\end{array}$} \\
\hline Sim & 917 & 46,8 & 17,0 & 1 & & 1 & \\
\hline Não & 1708 & 53,2 & 24,3 & $1,42(1,16-1,74)$ & 0,001 & $1,35(1,11-1,66)$ & 0,009 \\
\hline \multicolumn{8}{|c|}{ Consumo de frango com pele } \\
\hline Sim & 780 & 28,2 & 15,7 & 1 & & 1 & \\
\hline Não & 1845 & 71,8 & 22,9 & $1,45(1,14-1,85)$ & 0,002 & $1,42(1,12-1,81)$ & 0,007 \\
\hline \multicolumn{8}{|l|}{ Consumo de refrigerante } \\
\hline $\operatorname{Sim}$ & 723 & 28,4 & 10,3 & 1 & & 1 & \\
\hline Não & 1902 & 71,6 & 25,1 & $2,45(1,88-3,19)$ & $<0,01$ & $2,42(1,85-3,18)$ & $<0,01$ \\
\hline \multicolumn{8}{|c|}{ Consumo de leite integral } \\
\hline Sim & 1401 & 54,1 & 19,4 & 1 & & 1 & \\
\hline Não & 1224 & 45,9 & 22,7 & $1,17(0,96-1,43)$ & 0,115 & $1,14(0,94-1,39)$ & 0,160 \\
\hline \multicolumn{8}{|l|}{ Consumo de doces } \\
\hline Sim & 414 & 16,9 & 21,9 & 1 & & 1 & \\
\hline Não & 2211 & 83,1 & 20,7 & $0,94(0,73-1,22)$ & 0,664 & $0,97(0,75-1,25)$ & 0,759 \\
\hline \multicolumn{8}{|c|}{ Estado de saúde autoreferido } \\
\hline Ruim/Muito Ruim & 78 & 3,5 & 13,2 & 1 & & 1 & \\
\hline Regular & 641 & 21,8 & 15,4 & $1,17(0,34-3,94)$ & 0,804 & $1,07(0,30-3,09)$ & 0,943 \\
\hline Bom & 1276 & 49,9 & 19,1 & $1,44(0,43-4,79)$ & 0,551 & $1,30(0,37-4,55)$ & 0,714 \\
\hline Muito Bom & 610 & 24,8 & 30,3 & $2,29(0,69-7,63)$ & 0,176 & $1,91(0,54-6,71)$ & 0,323 \\
\hline \multicolumn{8}{|l|}{ Classificação do peso } \\
\hline Baixo peso/Eutrófico & 1183 & 49,8 & 20,0 & 1 & & 1 & \\
\hline Sobrepeso & 1084 & 37,4 & 23,1 & $1,15(0,94-1,42)$ & 0,176 & $1,12(0,91-1,38)$ & 0,411 \\
\hline Obesidade & 358 & 12,8 & 28,1 & $0,90(0,65-1,25)$ & 0,551 & $0,91(0,64-1,27)$ & 0,523 \\
\hline
\end{tabular}

${ }^{*}$ RP ajustada por idade e escolaridade.

mulheres com idade $\geq 35$ anos e homens com idade $\geq 65$ anos. O mesmo achado foi encontrado em outros países, sendo que o consumo estimado de frutas e hortaliças foi maior nos indivíduos mais velhos em 14 regiões geográficas, incluindo a África, América, Europa e Ásia ${ }^{10}$. No Brasil, vários estudos verificaram a influência positiva da idade sobre o consumo de frutas, legumes e verduras em ambos os $\operatorname{sexos}^{21,29,30}$. Em Ribeirão Preto/SP, Mondini et al. ${ }^{31}$ observaram que entre os homens, o consumo adequado de FLV associou-se à idade, sendo 2,8 vezes maior entre os idosos, quando comparados aos adultos entre 30 e 39 anos. No grupo de mulheres, o atendimento à recomendação da OMS foi 1,57 vezes maior entre aquelas com 50-59 anos quando comparadas às de $30 \mathrm{a} 39$ anos.
Esta associação positiva entre idade e consumo de frutas, legumes e verduras pode estar relacionada com as diferenças na formação do hábito alimentar entre as gerações. Indivíduos mais velhos formaram seu hábito alimentar durante um período menos marcante do consumo de alimentos processados, de alto teor de gorduras, açúcares e sal. Outro fator relacionado é o cuidado com a saúde e seguimento das orientações recebidas nos serviços de saúde entre pessoas de maior idade, que os procuram mais devido ao aumento da prevalência de doenças crônicas não transmissíveis nessa fase da vida ${ }^{32}$.

O estado civil, variável relacionada ao suporte social, tem sido mencionado como fator impactante no comportamento alimentar. Dietas saudáveis, principalmente aquelas que incluem 
Tabela 4. Prevalência de consumo recomendado de frutas, legumes e verduras (P\%), razão de prevalência bruta (RP) e ajustada (RPaj) e intervalo de confiança de 95\% (IC 95\%), segundo variáveis do estilo de vida e relacionadas à saúde para o sexo feminino, Região Centro-Oeste, VIGITEL, 2012.

\begin{tabular}{|c|c|c|c|c|c|c|c|}
\hline Variáveis & $\mathbf{n}$ & $\%$ & $\mathbf{P}(\%)$ & RP (IC 95\%) & p-valor & $\operatorname{RPaj}(\operatorname{IC} 95 \%)^{*}$ & p-valor \\
\hline \multicolumn{8}{|c|}{$\begin{array}{l}\text { Consumo de } \\
\text { bebida alcoólica }\end{array}$} \\
\hline Sim & 1034 & 26,6 & 30,8 & 1 & & 1 & \\
\hline Não & 3037 & 73,4 & 31,3 & $0,98(0,85-1,14)$ & 0,824 & $1,07(0,93-1,23)$ & 0,390 \\
\hline \multicolumn{8}{|c|}{$\begin{array}{l}\text { Consumo abusivo } \\
\text { de bebidas } \\
\text { alcoólicas }\end{array}$} \\
\hline Sim & 404 & 11,7 & 29,5 & 1 & & 1 & \\
\hline Não & 3667 & 88,3 & 31,4 & $1,06(0,86-1,32)$ & 0,568 & $1,09(0,89-1,34)$ & 0,475 \\
\hline \multicolumn{8}{|c|}{$\begin{array}{l}\text { Exercício Físico ( } 3 \\
\text { meses) }\end{array}$} \\
\hline Não & 2195 & 54,0 & 23,1 & 1 & & 1 & \\
\hline $\operatorname{Sim}$ & 1876 & 46,0 & 40,7 & $1,76(1,55-2,00)$ & $<0,01$ & $1,65(1,46-1,88)$ & $<0,01$ \\
\hline \multicolumn{8}{|c|}{$\begin{array}{l}\text { Atividade física no } \\
\text { lazer/transporte }\end{array}$} \\
\hline Não & 2538 & 61,1 & 25,4 & 1 & & 1 & \\
\hline Sim & 1533 & 38,9 & 40,2 & $1,58(1,40-1,78)$ & $<0,01$ & $1,50(1,33-1,70)$ & $<0,01$ \\
\hline \multicolumn{8}{|l|}{ Fuma } \\
\hline Sim & 345 & 8,3 & 23,1 & 1 & & 1 & \\
\hline Não & 3726 & 91,7 & 31,9 & $1,38(1,03-1,84)$ & 0,029 & $1,30(0,97-1,74)$ & 0,067 \\
\hline \multicolumn{8}{|l|}{ Cigarros/dia } \\
\hline $1-4$ & 44 & 16,4 & 16,8 & 1 & & 1 & \\
\hline $5-9$ & 51 & 17,0 & 22,0 & $1,31(0,42-4,07)$ & 0,640 & $1,33(0,45-4,08)$ & 0,775 \\
\hline $10-19$ & 99 & 33,7 & 22,3 & $1,33(0,44-4,00)$ & 0,615 & $1,37(0,47-4,04)$ & 0,750 \\
\hline$\geq 20$ & 91 & 32,9 & 18,4 & $1,09(0,33-3,57)$ & 0,883 & $1,11(0,38-4,01)$ & 0,910 \\
\hline
\end{tabular}

o consumo de FLV, são mais comumente observadas entre pessoas casadas ou que vivem com outros, especialmente entre os homens ${ }^{28}$. Estudos realizados nas populações brasileira, britânica e finlandesa verificaram que os homens casados consumiam mais frutas e hortaliças quando comparados àqueles sem companheiras ${ }^{24,31,33}$. No presente estudo, essa associação foi encontrada apenas no sexo feminino. Por outro lado, Campos et al. ${ }^{23}$, estudando a população adulta de Florianópolis, encontraram maior prevalência de consumo adequado de FLV entre indivíduos casados, de ambos os sexos.

Neste estudo, a prática de atividades físicas nos últimos 3 meses ou no lazer/transporte estiveram positivamente associadas à maior frequência de consumo de FLV em ambos os sexos. Em estudos nacionais ${ }^{23,34}$ e internacionais ${ }^{28,35,36}$ foram observados resultados similares. No Brasil, Campos et al. ${ }^{23}$ mostraram, em ambos os sexos, associação do consumo recomendado de FLV com atividade física no lazer. Já Figueiredo et al. ${ }^{37}$ verificaram entre os homens adultos da cidade de São Paulo, uma relação positiva entre a prática de atividade física no lazer e a maior frequência de consumo de FLV. A prática de atividade física regular associada com uma dieta saudável vem sendo amplamente divulgada como fator benéfico para a saúde. Estudos sugerem um efeito conjunto entre dieta rica em FLV e prática de atividade física na prevenção e tratamento da dislipdemia, hipertensão arterial, diabetes e osteoporose. Ambos são comportamentos modificáveis e a ausência ou presença de um tende a influenciar o outro, o que justifica o planejamento de ações conjuntas na promoção da saúde da população ${ }^{34}$. 
Tabela 4. continuação

\begin{tabular}{|c|c|c|c|c|c|c|c|}
\hline Variáveis & $\mathbf{n}$ & $\%$ & $\mathbf{P}(\%)$ & RP (IC 95\%) & p-valor & $\operatorname{RPaj}(\mathrm{IC} 95 \%)^{\star}$ & p-valor \\
\hline \multicolumn{8}{|l|}{ Consumo de feijão } \\
\hline Não & 1106 & 24,1 & 30,3 & 1 & & 1 & \\
\hline $\operatorname{Sim}$ & 2965 & 75,9 & 31,4 & $1,04(0,90-1,19)$ & 0,618 & $1,12(0,98-1,29)$ & 0,083 \\
\hline \multicolumn{8}{|c|}{$\begin{array}{l}\text { Consumo carne com excesso de } \\
\text { gordura }\end{array}$} \\
\hline Sim & 749 & 27,9 & 24,1 & 1 & & 1 & \\
\hline Não & 3322 & 72,1 & 33,9 & $1,41(1,20-1,65)$ & $<0,01$ & $1,34(1,14-1,57)$ & 0,001 \\
\hline \multicolumn{8}{|c|}{ Consumo de frango com pele } \\
\hline Sim & 588 & 15,1 & 24,1 & 1 & & 1 & \\
\hline Não & 3483 & 84,9 & 32,4 & $1,35(1,09-1,67)$ & 0,007 & $1,26(1,02-1,56)$ & 0,047 \\
\hline \multicolumn{8}{|l|}{ Consumo de refrigerante } \\
\hline Sim & 778 & 22,4 & 18,4 & 1 & & 1 & \\
\hline Não & 3293 & 77,6 & 34,8 & $1,89(1,51-2,35)$ & $<0,01$ & $1,81(1,45-2,26)$ & $<0,01$ \\
\hline \multicolumn{8}{|l|}{ Consumo de leite integral } \\
\hline Sim & 2071 & 51,7 & 28,8 & 1 & & 1 & \\
\hline Não & 2000 & 48,3 & 33,7 & $1,17(1,03-1,32)$ & 0,014 & $1,12(0,99-1,26)$ & 0,097 \\
\hline \multicolumn{8}{|l|}{ Consumo de doces } \\
\hline Sim & 672 & 18,2 & 35,6 & 1 & & 1 & \\
\hline Não & 3399 & 81,8 & 30,2 & $0,85(0,73-0,99)$ & 0,033 & $0,89(0,77-1,04)$ & 0,155 \\
\hline \multicolumn{8}{|c|}{ Estado de saúde autoreferido } \\
\hline Ruim/Muito Ruim & 240 & 5,8 & 19,4 & 1 & & 1 & \\
\hline Regular & 1228 & 29,6 & 25,6 & $1,32(0,91-1,91)$ & 0,136 & $1,30(0,90-1,87)$ & 0,132 \\
\hline Bom & 1776 & 44,3 & 30,9 & $1,59(1,12-2,28)$ & 0,010 & $1,48(1,04-2,10)$ & 0,023 \\
\hline Muito Bom & 786 & 20,3 & 42,8 & $2,21(1,54-3,17)$ & $<0,01$ & $1,93(1,35-2,78)$ & $<0,01$ \\
\hline \multicolumn{8}{|l|}{ Classificação do Peso } \\
\hline Baixo peso/Eutrófico & 2218 & 56,1 & 32,0 & 1 & & 1 & \\
\hline Sobrepeso & 1348 & 30,4 & 31,2 & $0,97(0,85-1,12)$ & 0,722 & $0,97(0,84-1,11)$ & 0,574 \\
\hline Obesidade & 504 & 13,5 & 27,8 & $0,87(0,71-1,06)$ & 0,170 & $0,93(0,76-1,13)$ & 0,346 \\
\hline
\end{tabular}

* RP ajustada para idade e escolaridade.

Dentre as variáveis comportamentais, o tabagismo mostrou associação limítrofe com o desfecho avaliado para ambos os sexos e o consumo abusivo de bebidas alcoólicas apresentou associação marginal para o sexo masculino, na análise múltipla. É possível que a baixa prevalência de fumantes e de consumidores abusivos de bebidas alcoólicas tenha contribuído para esse resultado. Outros estudos mostraram a associação do consumo adequado de FLV e o hábito de não fumar e de não consumir bebidas alcoólicas ${ }^{23,30}$. Este fato pode revelar características comportamentais dos consumidores de FLV, como a preocupação com a saúde e a existência de um comportamento protetor.

As variáveis de consumo alimentar, representadas por alimentos pertencentes a um padrão alimentar não saudável, como carne com excesso de gordura, frango com pele e o consumo de refrigerantes e/ou sucos artificiais apresentaram associação negativa com o recomendado de FLV. Resultados semelhantes foram encontrados em um estudo realizado na cidade de Bélem $(\mathrm{PA})^{22}$, onde a frequência de consumo recomendado de FLV foi aproximadamente 2,8 vezes maior entre homens que não consumiam refrigerantes e 13,3 vezes maior entre as mulheres que não comiam carne com excesso de gordura ou frango com pele. Outros estudos mostraram que alimentos ricos em gorduras e açúcares estão inversamente relacionados ao consumo de frutas, legumes e verduras ${ }^{37,38}$. Essa associação pode ser um reflexo da preocupação dos indivíduos com a sua saúde, pois os mais conscientes do seu comportamento alimentar são, normalmente, aqueles que buscam maiores informações sobre saúde e alimentação, refletindo nas suas escolhas e atitudes alimentares. 
Neste estudo, o consumo regular de feijão apresentou associação com o consumo recomendado de FLV para os homens e associação marginal para as mulheres. É possível que a associação encontrada seja compatível com a hipótese da coexistência de hábitos alimentares saudáveis, como tem sido sugerido na literatura ${ }^{37}$. Segundo a Pesquisa de Orçamentos Familiares (POF) 2008-2009, a Região Centro-Oeste apresentou consumo médio per capita de feijão maior que as demais regiões ${ }^{39}$. Vale ressaltar que o consumo desse alimento está muito relacionado ao hábito alimentar brasileiro e pode não ser um bom marcador de alimentação saudável globalmente.

Os fatores associados ao consumo recomedado de FLV foram, em sua maioria, relacionados ao estilo de vida, evidenciando uma tendência de agregação de comportamentos que identificam um padrão de vida saudável. Este resultado é interessante, pois corrobora a hipótese de que comportamentos que potenciam a saúde tendem a agrupar-se, porém deve-se reforçar a ideia de que alimentação saudável é um fenômeno complexo e multifacetado ${ }^{40,41}$.

Com relação ao estado de saúde autorreferido, verificou-se que a prevalência de consumo recomendado FLV foi maior entre as mulheres que referiram seu estado de saúde como bom ou muito bom. Resultados semelhantes foram encontrados em outros estudos no Brasil ${ }^{23,42}$. Na Europa, o consumo adequado de FLV foi associado a indicadores autorreferidos de boa saúde física funcional ${ }^{43}$. Esta variável é considerada um indicador adequado e essencial do estado de saúde de indivíduos e de populações, compondo um preditor poderoso da mortalidade, independentemente de fatores médicos, comportamentais e psicossociais $^{44}$.

A variável classificação do peso não mostrou associação com o consumo de FLV. Entretanto, estudos verificaram associação direta entre o consumo de FLV e o excesso de peso ${ }^{22,43}$. Sichieri e Moura, analisando as variações do índice de massa corporal entre adultos no Brasil, observaram que o consumo de FLV não se associou com IMC de mulheres e sim positivamente em homens, sugerindo que somente o aumento no consumo desses alimentos não é capaz de deslocar o consumo de alimentos mais calóricos e menos saudáveis, que contribuem para o aumento do $\mathrm{IMC}^{45}$. Indivíduos com excesso de peso podem apresentar relação positiva com o consumo adequado de FLV, significando uma maior preocupação com a recuperação da saúde, além da tentativa de redução de peso através de uma dieta mais saudável ${ }^{23}$.
Os resultados obtidos neste estudo, em sua maioria, são consistentes com os dados encontrados na literatura, porém algumas limitações precisam ser consideradas. Uma delas é o possível viés de seleção, visto que o VIGITEL não incluiu indivíduos residentes em domicílios sem telefone fixo. Embora o fator de expansão utilizado para correção das frequências das variáveis estudadas leve em consideração toda a população, este estudo cobre apenas a população com telefone residencial, o que pode levar a uma superestimação do consumo de frutas, legumes e verduras ${ }^{46}$.

Outra limitação refere-se às diferenças entre os métodos para avaliar, definir e categorizar a frequência de consumo de FLV que podem prejudicar a comparação com outros estudos sobre consumo populacional desses alimentos. No sistema VIGITEL, as perguntas referem-se à frequência de consumo (número de vezes ao dia) e não à quantidade (gramas ou porções) de frutas e hortaliças consumida no dia, como proposto pela OMS. Esta diferença na medida do consumo pode levar a erros de classificação dos indivíduos para este indicador. Ainda assim, a adoção do indicador "frequência de consumo" de frutas e hortaliças, independente da especificação da porção, é bastante comum na literatura mundial ${ }^{4,9}$.

Dentre as vantagens desta modalidade de inquérito destaca-se a maior agilidade e baixo custo na obtenção das informações a partir de uma amostra representativa da população, permitindo sua realização de forma sistemática ${ }^{47}$.

Pode-se destacar a contribuição deste estudo por meio da análise de dados comparáveis, descrevendo a importância e a distribuição de um comportamento alimentar reconhecido como protetor para DCNT em uma região do país com baixa produção científica sobre o assunto. As informações contidas no presente estudo revelam desafios importantes para pesquisadores e gestores, podendo fornecer subsídios para ações de políticas públicas que visem à promoção e à manutenção da alimentação saudável.

\section{Considerações finais}

Embora o conhecimento e a divulgação de dados nacionais sobre fatores de risco e proteção para as DCNT sejam imprescindíveis para a tomada de um conjunto de medidas de controle dessas doenças, a geração de informações com base científica sobre a distribuição desses fatores nas diferentes regiões do país desempenha um papel fundamental no enfrentamento dos desafios atu- 
ais, auxiliando na definição de prioridades e organização de ações de saúde mais impactantes, de acordo com as necessidades de cada região. Com relação à alimentação, o plano de enfrentamento das doenças crônicas prevê mecanismos de aumento da produção de FLV, visando à redução de custos, contribuindo para o acesso a alimentos saudáveis.

\section{Colaboradores}

TF Damiani participou do desenvolvimento do estudo, análise estatística, interpretação dos dados e redação do manuscrito. LP Pereira participou da análise e interpretação de resultados e revisão crítica do manuscrito. MG Ferreira participou do desenho e coordenação do estudo, análise estatística, interpretação dos dados e redação do manuscrito.

\section{Agradecimentos}

Os autores agradecem ao Professor Doutor Neuber José Segri, da Universidade Federal de Mato Grosso-MT, pelo auxílio na análise dos dados e à professora Doutora Deborah Carvalho Malta, da Secretaria de Vigilância em Saúde - Brasília/ DF, pela disponibilização do banco de dados para análise. 


\section{Referências}

1. Instituto Brasileiro de Geografia e Estatística (IBGE). Diretoria de Pesquisas. Síntese de Indicadores 2012. Pesquisa Nacional por Amostra de Domicílios. Rio de Janeiro: IBGE; 2013.

2. Programa das Nações Unidas para o Desenvolvimento (PNUD). Atlas do desenvolvimento humano no Brasil: $O$ Indice de Desenvolvimento Humano Municipal Brasileiro. Brasilia: PNUD; 2013

3. Instituto de Pesquisa Econômica Aplicada (IPEA). Política de comercialização agrícola no Brasil: Estado, planejamento e políticas públicas. Brasília: IPEA; 2010.

4. Brasil. Ministério da Saúde (MS). VIGITEL Brasil 2012. Vigilância de fatores de risco e proteção para doenças crônicas por inquérito telefônico. Brasília: MS; 2013.

5. Organização Pan-Americana de Saúde (OPAS), Organização Mundial da Saúde (OMS). Doenças crônico-degenerativas e obesidade: estratégia mundial sobre alimentação saudável, atividade física e saúde. Brasília: OPAS, OMS; 2003.

6. World Health Organization (WHO). Diet, nutrition and the prevention of chronic diseases: report of a joint WHO/FAO expert consultation. Geneva: WHO; 2003.

7. Rolls BJ, Ello-Martin JA, Tohill BC. What can intervention studies tell us about the relationship between fruit and vegetable consumption and weight management? Nutr Rev 2004; 62(1):1-17.

8. Brasil. Ministério da Saúde (MS). Secretaria de atenção à Saúde. Coordenação-Geral da política de Alimentação e Nutrição. Guia Alimentar para a População Brasileira (versão para consulta pública). Brasília: MS; 2014.

9. Blanck HM, Gillespie C, Kimmons JE, Seymour JD, Serdula MK. Trends in fruit and vegetable consumption among U.S. men and women, 1994-2005. Prev Chronic Dis. 2008; 5(2):A35.

10. Lock K, Pomerleau J, Causer L, Altmann DR, McKee M. The global burden of disease attributable to low consumption of fruit and vegetables: implications for the global strategy on diet. Bull World Health Organ 2005; 83(2):100-108.

11. Levy-Costa RB, Sichieri R, Pontes NS, Monteiro CA. Disponibilidade domiciliar de alimentos no Brasil: distribuição e evolução (1974-2003). Rev Saude Publica 2005; 39(4):530-540.

12. Instituto Brasileiro de Geografia e Estatística (IBGE). Pesquisa de Orçamentos Familiares 2008-2009: Mais de $90 \%$ da população comem poucas frutas, legumes e verduras. Rio de Janeiro: Instituto Brasileiro de Geografia e Estatística; 2010.

13. Moura EC, Morais Neto OL, Malta DC, Moura L, Silva NN, Bernal R, Claro RM, Monteiro CA. Vigilância de Fatores de Risco para Doenças Crônicas por Inquérito Telefônico nas capitais dos 26 estados brasileiros e no Distrito Federal (2006). Rev Bras Epidemiol 2008; 11(Supl. 1):20-37.

14. World Health Organization (WHO). Global recommendations on physical activity for health. Geneva: WHO; 2010 .

15. World Health Organization (WHO). Expert Committee: physical status: the use and interpretation of anthropometry. Geneva: WHO; 1995. (Technical Report Series, n. 854)

16. World Health Organization (WHO). Obesity: preventing and managing the global epidemic. Geneva: WHO; 1998.
17. Cervi A, Franceschni SCC, Priore SE. Análise crítica do uso do índice de massa corporal para idosos. Rev Nutr 2005; 18(6):765-775.

18. Centers Diseases Controls and Prevention (CDC). Prevalence and Trends Data: Behavioral Risk Factor Surveillance System. Atlanta: CDC; 2009.

19. Centers for Disease Control and Prevention (CDC). Strategies to Prevent Obesity and Other Chronic Diseases: The CDC Guide to Strategies to Increase the Consumption of Fruits and Vegetables. Atlanta: U.S. Department of Health and Human Services; 2011. [acessado 2014 ago 5]. Disponível em: http://www.cdc. gov/obesity/resources/recommendations.html

20. Taxa de prevalência do consumo recomendado de frutas e verduras, por ano, segundo região e escolaridade [Internet]. Rio de Janeiro: Portal Determinantes Sociais da Saúde. Observatório sobre Iniquidades em Saúde. CEPI-DSS/ENSP/FIOCRUZ; 2012. [acessado 2014 ago 8]. Disponível em: http://dssbr.org/site/wpcontent/uploads/2012/03/Ind010403-20120130.pdf

21. Fisberg RM, Morimoto JM, Slater B, Barros MB, Carandina L, Goldbaum M, Oliveira Latorre MR, César CL. Dietary quality and associated factors among adults living in the state of Sao Paulo, Brazil. J Am Diet Assoc 2006; 106(12):2067-2072.

22. Moura EC, Dias RM, Reis RC. Determinantes do consumo de frutas, legumes e verduras na população adulta de Belém, Pará, 2005. Nutrire Rev Soc Bras Aliment Nutr 2007; 32(2):29-40.

23. Campos VC, Bastos JL, Gauche H, Boing AF, Assis MAA. Fatores associados ao consumo adequado de frutas, legumes e verduras em adultos de Florianópolis. Rev Bras Epidemiol 2010; 13(2):352-362.

24. Billson H, Pryer JA, Nichols R. Variation in fruit and vegetable consumption among adults in Britain. An analysis from the dietary and nutritional survey of British adults. Eur J Clin Nutr 1999; 53(12):946-952.

25. Instituto de Pesquisa Econômica Aplicada (IPEA). Situação Social nos Estados: o caso do Distrito Federal. Brasília: IPEA; 2012.

26. Turrell G, Hewitt B, Patterson C, Oldenburg B. Measuring socio-economic position in dietary research: is choice of socio-economic indicator important? Public Health Nutr 2003; 6(2):191-200.

27. Thompson FE, Midthune D, Subar AF, McNeel T, Berrigan D, Kipnis V. Dietary intake estimates in the National Health Interview Survey, 2000: methodology, results, and interpretation. J Am Diet Assoc 2005; 105(3):352-363

28. Estaquio C, Druesne-Pecollo N, Latino-Martel P, Dauchet L, Hercberg S, Bertrais S. Socioeconomic differences in fruit and vegetable consumption among middle-aged French adults: adherence to the 5 a day recommendation. J Am Diet Assoc 2008; 108(12):20212030.

29. Jaime PC, Monteiro CA. Fruit and vegetable intake by Brazilian adults, 2003. Cad Saude Publica 2005; 21(Supl. 1):S19-24.

30. Neutzling MB, Rombaldi AJ, Azevedo MR, Hallal PC. Fatores associados ao consumo de frutas, legumes e verduras em adultos de uma cidade no Sul do Brasil. Cad Saude Publica 2009; 25(11):2365-2374. 
31. Mondini L, Moraes SA, Freitas ICM, Gimeno SGA. Consumo de frutas e hortaliças por adultos em Ribeirão Preto, SP. Rev Saude Publica 2010; 44(4):686-694.

32. Jaime PC, Figueiredo ICR, Moura EC, Malta DC. Fatores associados ao consumo de frutas e hortaliças no Brasil, 2006. Rev Saúde 2009; 43(2):57-64.

33. Prättälä R, Paalanen L, Grinberga D, Helasoja V, Kasmel A, Petkeviciene J. Gender differences in the consumption of meat, fruit and vegetables are similar in Finland and Baltic countries. Eur J Public Health 2007; 17(5):520-525.

34. Tassitano RM, Tenório MCM, Cabral PC, Silva GAP. Clustering of physical inactivity and low fruit and vegetables intake and associated factors in young adults. Rev. Nutr. 2014; 27(1):25-44.

35. Jago R, Nicklas T, Yang SJ, Baranowski T, Zakeri I, Berenson GS. Physical activity and health enhancing dietary behaviors in young adults: Bogalusa Heart Study. Prev Med 2005; 41(1):194-202.

36. Camoes M, Lopes C. Dietary intake and different types of physical activity: full-day energy expenditure, occupational and leisure-time. Public Health Nutr 2008; 11(8):841-848.

37. Figueiredo ICR, Jaime PC, Monteiro CA. Fatores associados ao consumo de frutas, legumes e verduras em adultos da cidade de São Paulo. Rev Saude Publica 2008; 42(5):777-785.

38. Forshee RA, Storey ML. The role of added sugars in the diet quality of children and adolescents. J AM Coll Nutr 2001; 20(1):32-43.

39. Instituto Brasileiro de Geografia e Estatística (IBGE). Pesquisa de Orçamentos Familiares - POF 2008-2009. Análise do consumo alimentar pessoal no Brasil. Rio de Janeiro: IBGE; 2011.

40. Mota J, Sallis J. Actividade física e saúde. Factores de influência da actividade física nas crianças e nos adolescentes. Porto: Campo de Letras; 2002.

41. Palma RFM, Barbieri P, Damião R, Poletto J, Chaim R, Gimeno SG, Ferreira SRG, Sartorelli DS; Grupo de Estudos em Diabetes na População Nipo-Brasileira de Bauru, SP. Fatores associados ao consumo de frutas, verduras e legumes em Nipo-Brasileiros. Rev Bras Epidemiol 2009; 12(3):436-445.
42. Barros, MBA, Zanchetta LM, Moura EC, Malta DC Auto-avaliação da saúde e faotres associados, Brasil. 2006. Rev Saude Publica 2009; 43(2):27-37.

43. Myint PK, Welch AA, Bingham SA, Surtees PG, Wainwright NW, Luben RN, Wareham NJ, Smith RD, Harvey IM, Day NE, Khaw KT. Fruit and vegetable consumption and self-reported functional health in men and women in the European Prospective Investigation into Cancer-Norfolk (EPIC-Norfolk):a population-based cross-sectional study. Public health nutr 2007; 10(1):34-41.

44. Idler EL, Benyamini Y. Self-rated health and mortality: a review of twenty-seven community studies. $J$ Health Soc Behav 1997; 38(1):21-37.

45. Sichieri R., Moura ECD. Análise multinível das variações no índice de massa corporal entre adultos, Brasil, 2006. Rev Saude Publica 2009; 43(2):90-97.

46. Monteiro CA, Moura EC, Jaime PC, Claro RM. Validade de indicadores do consumo de alimentos e bebidas obtidos por inquérito telefônico. Rev Saude Publica 2008; 42(4):582-589.

47. Carvalhaes MABL, Moura EC, Monteiro CA. Prevalência de fatores de risco para doenças crônicas: inquérito populacional mediante entrevistas telefônicas em Botucatu, São Paulo, 2004. Rev Bras Epidemiol 2008; 11(1):14-23

Artigo apresentado em 05/07/2015

Aprovado em 27/11/2015

Versão final apresentada em 29/11/2015 